PROCEEDINGS OF THE

AMERICAN MATHEMATICAL SOCIETY

Volume 133, Number 8, Pages 2225-2231

S 0002-9939(05)08004-

Article electronically published on March 17, 2005

\title{
POLYNOMIAL GAUSS SUMS
}

STEPHEN D. COHEN, MICHAEL DEWAR, JOHN B. FRIEDLANDER, DANIEL PANARIO, AND IGOR E. SHPARLINSKI

(Communicated by Wen-Ching Winnie Li)

\begin{abstract}
A recent bound for exponential sums by Friedlander, Hansen and Shparlinski is extended to twisted exponential sums with general polynomial arguments. As a by-product a new result about perfect powers in certain products of polynomials is established.
\end{abstract}

\section{INTRODUCTION}

Denote by $\mathbb{Z}_{t}$ the residue ring modulo $t$ and by $\mathcal{U}_{t}$ its group of units, that is, the collection of residue classes which are relatively prime to $t$. A sequence $\mathcal{Z}=\left(z_{1}, \ldots, z_{T}\right)$ of $T$ elements from $\mathbb{Z}_{t}$ is called $\mathcal{K}$-invariant if $\mathcal{K} \subseteq \mathcal{U}_{t}$ is such that the sequence $k z_{1}, \ldots, k z_{T}$, taken modulo $t$, is a permutation of the original sequence $z_{1}, \ldots, z_{T}$ for each $k \in \mathcal{K}$.

For a prime $p$ and an integer $a$, define the additive character

$$
\psi(z)=\exp (2 \pi i a z / p)
$$

of $\mathbb{F}_{p}$. We also use $\chi(z)$ to denote a multiplicative character of $\mathbb{F}_{p}^{*}$, extended to the whole field $\mathbb{F}_{p}$ by setting $\chi(0)=0$. As usual $\chi$ is said to be of order $s$ if $s$ is the smallest positive integer for which $\chi^{s}=\chi_{0}$, the trivial multiplicative character. (We refer to [5] for an exhaustive description of multiplicative and additive characters, and other important notions of the theory of finite fields.)

Let $\vartheta$ be an integer of multiplicative order $t \geq 1$ modulo $p$. Given two polynomials $f(X), g(X) \in \mathbb{F}_{p}[X]$ and additive and multiplicative characters $\psi$ and $\chi$ over $\mathbb{F}_{p}$, we define the twisted exponential sum

$$
S_{\mathcal{Z}}(\chi, \psi ; f, g)=\sum_{z_{s} \in \mathcal{Z}} \chi\left(f\left(\vartheta^{z_{s}}\right)\right) \psi\left(g\left(\vartheta^{z_{s}}\right)\right)
$$

Received by the editors October 20, 2003.

2000 Mathematics Subject Classification. Primary 11L07, 11T23; Secondary 11B50, 11K31.

The second author was supported in part by an NSERC Undergraduate Student Research Award.

The third author was supported in part by NSERC grant A5123 and a Killam Research Fellowship.

The fourth author was supported in part by NSERC grant 238757 .

The fifth author was supported in part by ARC grant A69700294. 
over a $\mathcal{K}$-invariant set $\mathcal{Z}$ for some set $\mathcal{K}$. (Of course, this sum also depends on $\vartheta$ but perhaps the notation is already sufficiently complicated.)

In the special case that $f(X)=g(X)=X$, if $\vartheta$ is a primitive root and $\mathcal{Z}=\mathcal{U}_{p}$, this is simply the Gauss sum. Further, when $g(X)=X$ and $f(X)=1$, such sums have been estimated in [2, 3. Here that method is modified to yield bounds for the more general sums $S_{\mathcal{Z}}(\chi, \psi ; f, g)$. We remark that when the additive character influences the sum in a nontrivial way, then these sums can be treated in exactly the same way as the sums from [2, 3]. However, in the other case, that is, when either $\psi$ is trivial or $g(X)$ is constant, one needs to use some new arguments to establish the applicability of the Weil bound. We anticipate that the ensuing result (Lemma 1) may have some other applications.

\section{Preliminaries}

We start with the following statement which may be of independent interest.

Lemma 1. Let $s>1$ be an integer with $\operatorname{gcd}(s, p)=1$. Also, for some $m \geq 1$, let $\left\{k_{1}, \ldots, k_{m}\right\}$ be a set of $m$ distinct positive integers with $\operatorname{gcd}\left(k_{j}, p\right)=1$ for each $j \leq m$ and let $\left\{d_{1}, \ldots, d_{m}\right\}$ be a further set of positive integers. Suppose that $f(X) \in \mathbb{F}_{p}[X]$ is a polynomial indivisible by $X$. We define

$$
F(X)=\prod_{j=1}^{m} f\left(X^{k_{j}}\right)^{d_{j}} .
$$

If $F(X)$ is an s-th power of a polynomial in $\mathbb{F}_{p}[X]$, then there is a factorisation $s=s_{0} d_{0}$ and a polynomial $f_{0}(X) \in \mathbb{F}_{p}[X]$ such that $f(X)=f_{0}(X)^{s_{0}}$ and $d_{0}$ divides $d_{j}$ for each $j \leq m$.

Proof. The proof proceeds by induction on $s$. First it is established for $s$ prime, and then, using induction, for $s$ composite.

Assume first that $s$ is prime. Without loss of generality we can suppose that the standard decomposition of $f(X)$ in terms of distinct polynomials, irreducible over $\mathbb{F}_{p}[X]$, can be expressed as

$$
f(X)=\prod_{i=1}^{r} f_{i}(X)^{t_{i}}, \quad 1 \leq t_{i}<s, i=1, \ldots, r,
$$

and also that $1 \leq d_{j}<s, j=1, \ldots, m$. Since the $k_{1}, \ldots, k_{m}$ are distinct, we can also suppose that $k_{1}>\ldots>k_{m}$. Moreover, let $e_{i}$ be the order of the irreducible polynomial $f_{i}(X), 1 \leq i \leq r$. Because polynomials $f_{i}(X)$ are irreducible, this is also the order of each of the roots of $f_{i}, 1 \leq i \leq r$. We can suppose the latter are ordered so that $e_{1} \geq \ldots \geq e_{r}$.

Evidently, the order of any root (or irreducible factor) of $f_{i}\left(X^{k_{j}}\right), 1 \leq i \leq r$, $1 \leq j \leq m$, is a divisor of $k_{j} e_{i}$ (that is itself divisible by $e_{i}$ ). Certainly it does not exceed $k_{j} e_{i}$. Now, the result of Butler [1] implies, for example, that the polynomial $f_{1}\left(X^{k_{1}}\right)$ has at least one irreducible factor of order $k_{1} e_{1} / l$ for each divisor $l$ of $k_{1}$ relatively prime to $e_{1}$. Take $l=1$ to deduce the existence of an irreducible factor $g(X)$ of $f_{1}\left(X^{k_{1}}\right)$ of order precisely $k_{1} e_{1}>k_{j} e_{i}, i \geq 1, j>1$. Further, $f_{1}\left(X^{k_{1}}\right), \ldots, f_{r}\left(X^{k_{1}}\right)$ are pairwise relatively prime (since $f_{1}, \ldots, f_{r}$ are). It follows that the irreducible polynomial $g(X)$ is not a factor of any polynomial $f_{i}\left(X^{k_{j}}\right)$ 
except $f_{1}\left(X^{k_{1}}\right)$. Accordingly, $g$ appears in the product $F$ with precise multiplicity $t_{1} d_{1}$, and this is indivisible by $s$. The result for $s$ prime follows.

Now suppose $s$ is composite. Let $s_{0}$ be the maximal divisor of $s$ such that $f(X)=f_{0}(X)^{s_{0}}, f_{0}(X) \in \mathbb{F}_{p}[X]$. We can assume $s_{0} \neq s$ (otherwise the result is trivial). Further, if $s_{0}>1$, we can use induction applied to $f_{0}$ with $s$ replaced by $s / s_{0}$ to obtain the result. Hence we can suppose $s_{0}=1$.

Next, suppose $d_{0}=\operatorname{gcd}\left(d_{1}, \ldots, d_{m}, s\right)>1$. If $d_{0}=s$, then again the result is trivial. Further, if $1<d_{0}<s$, we replace $s$ by $s / d_{0}$ and each $d_{i}$ by $d_{i} / d_{0}$. Apply induction to conclude that $F$ cannot be an $s$-th power.

Consequently, we may suppose $s_{0}=d_{0}=1$. Now take any prime divisor $l$ of $s$ and set $w=s / l$. Thus $1<w<s$ (since $s$ is composite). Trivially, $f(X)=$ $g(X)^{u}$ for some polynomial $g(X) \in \mathbb{F}_{p}[X]$ and an integer $u \mid w$, only if $u=1$, and $\operatorname{gcd}\left(d_{1}, \ldots, d_{m}, w\right)=1$ (since the corresponding assertions hold for $s$ instead of $w$ ). By induction, with $s$ replaced by $w, F$ cannot be a $w$-th power and therefore cannot be an $s$-th power.

We now recall Lemma 2 from [2].

Lemma 2. Suppose that $\mathcal{K} \subseteq \mathcal{U}_{t}$ is a set of cardinality $|\mathcal{K}|=K$. Then, for any fixed $\delta>0$ and any integer $h \geq t^{\delta}$ there exists an integer $r \in \mathcal{U}_{t}$ such that the congruence

has

$$
r k \equiv y \quad(\bmod t), \quad k \in \mathcal{K}, 0 \leq y \leq h-1,
$$

$$
L_{r}(h) \gg \frac{K h}{t}
$$

solutions, where the implied constant depends on $\delta$.

We also need the Weil bound for character sums (see Theorem 3 of Chapter 6 of [4]).

Lemma 3. Let $F(X)$ be a non-zero rational function over $\mathbb{F}_{p}$ of degree $m$ and let $G(X)$ be polynomial over $\mathbb{F}_{p}$ of degree $n$. Let $\chi$ be a multiplicative character of $\mathbb{F}_{p}^{*}$ of order $s \geq 1$ and let $\psi$ be an additive character of $\mathbb{F}_{p}$. Assume that at least one of the following holds:

- either $\chi$ is non-trivial and $F$ is not an s-th power of a rational function over the algebraic closure of $\mathbb{F}_{p}$;

- or $\psi$ is non-trivial and $G$ is not constant modulo $p$.

Then

$$
\left|\sum_{x \in \mathbb{Z}_{p}} \chi(F(x)) \psi(G(x))\right| \leq(m+n-1) p^{1 / 2} .
$$

\section{MAin RESUlts}

The following bound 1 is our main result. It provides a direct generalization of Lemma 4 from [2].

Theorem 4. Let $\mathcal{Z}=\left(z_{1}, \ldots, z_{T}\right)$ be a $\mathcal{K}$-invariant sequence of elements of $\mathbb{Z}_{t}$ with respect to the set $\mathcal{K} \subseteq \mathcal{U}_{t}$ of cardinality $K=|\mathcal{K}|$ and let $N$ be the number of

\footnotetext{
${ }^{1}$ At the risk of stating the obvious we mention that, by an expression such as $N^{1 / 2 \nu}$, we mean $N^{1 /(2 \nu)}$ and not $N^{\nu / 2}$.
} 
solutions of the congruence $z_{\ell} \equiv z_{r}(\bmod t), 1 \leq \ell, r \leq T$. Let $f(X)$ and $g(X)$ be two polynomials over $\mathbb{F}_{p}$ each of degree at most $D$. Assume that at least one of the following holds:

- either the multiplicative character $\chi$ is non-trivial of order $s$ and $f$ is not an $s$-th power of a rational function over the algebraic closure of $\mathbb{F}_{p}$;

- or $\psi$ is non-trivial and $g$ is not constant modulo $p$.

If $t \geq p^{1 / 2+\varepsilon}$, then for any integer $\nu \geq 1$ the bound

$$
\left|S_{\mathcal{Z}}(\chi, \psi ; f, g)\right| \ll D^{1 / 2 \nu} N^{1 / 2 \nu} T^{1-1 / \nu} K^{-1 / 2(\nu+1)} t^{1 / 2 \nu} p^{1 / 4(\nu+1)}
$$

holds, where the implied constant depends on $\varepsilon$ and $\nu$.

Proof. Fix some $\varepsilon>0$ and put

$$
h=\left\lceil t K^{-\nu /(\nu+1)} p^{-1 / 2(\nu+1)}\right\rceil .
$$

In this case

$$
h \geq t^{1 /(\nu+1)} p^{-1 / 2(\nu+1)} \geq p^{\varepsilon /(\nu+1)},
$$

thus Lemma 2 applies.

We select $r$ as in Lemma 2 Let $\mathcal{L}$ denote the subset of $\mathcal{K}$ which satisfies the corresponding congruence and let $L=|\mathcal{L}|$.

Define $Q(x)$ as the number of elements $z \in \mathcal{Z}$ with $z \equiv x(\bmod t)$. Note that

$$
\sum_{x \in \mathbb{Z}_{t}} Q(x)=T
$$

and

$$
\sum_{x \in \mathbb{Z}_{t}} Q(x)^{2}=N
$$

We also have $Q(k x)=Q(x)$ for any $k \in \mathcal{K}$ since repetitions in $\mathcal{Z}$ are preserved under the permutation of $\mathcal{Z}$ generated by multiplication by $k \in \mathcal{K}$. Therefore

$$
\begin{aligned}
S_{\mathcal{Z}}(\chi, \psi ; f, g) & =\sum_{z_{s} \in \mathcal{Z}} \chi\left(f\left(\vartheta^{z_{s}}\right)\right) \psi\left(g\left(\vartheta^{z_{s}}\right)\right) \\
& =\sum_{x \in \mathbb{Z}_{t}} Q(x) \chi\left(f\left(\vartheta^{x}\right)\right) \psi\left(g\left(\vartheta^{x}\right)\right) \\
& =\frac{1}{L} \sum_{k \in \mathcal{L}} \sum_{x \in \mathbb{Z}_{t}} Q(k x) \chi\left(f\left(\vartheta^{k x}\right)\right) \psi\left(g\left(\vartheta^{k x}\right)\right) \\
& =\frac{1}{L} \sum_{k \in \mathcal{L}} \sum_{x \in \mathbb{Z}_{t}} Q(x) \chi\left(f\left(\vartheta^{k x}\right)\right) \psi\left(g\left(\vartheta^{k x}\right)\right) \\
& =\frac{1}{L} \sum_{x \in \mathbb{Z}_{t}} Q(x) \sum_{k \in \mathcal{L}} \chi\left(f\left(\vartheta^{k x}\right)\right) \psi\left(g\left(\vartheta^{k x}\right)\right) .
\end{aligned}
$$


Two applications of the Hölder inequality yield

$$
\begin{aligned}
& \left|S_{\mathcal{Z}}(\chi, \psi ; f, g)\right|^{2 \nu} \\
& \quad \leq L^{-2 \nu}\left(\sum_{x \in \mathbb{Z}_{t}} Q(x)\left|\sum_{k \in \mathcal{L}} \chi\left(f\left(\vartheta^{k x}\right)\right) \psi\left(g\left(\vartheta^{k x}\right)\right)\right|\right)^{2 \nu} \\
& \quad=L^{-2 \nu}\left(\sum_{x \in \mathbb{Z}_{t}}\left(Q(x)^{2}\right)^{1 / 2 \nu} Q(x)^{(\nu-1) / \nu}\left|\sum_{k \in \mathcal{L}} \chi\left(f\left(\vartheta^{k x}\right)\right) \psi\left(g\left(\vartheta^{k x}\right)\right)\right|\right)^{2 \nu} \\
& \quad \leq L^{-2 \nu} \sum_{x \in \mathbb{Z}_{t}} Q(x)^{2}\left(\sum_{x \in \mathbb{Z}_{t}} Q(x)\right)^{2 \nu-2} \sum_{x \in \mathbb{Z}_{t}}\left|\sum_{k \in \mathcal{L}} \chi\left(f\left(\vartheta^{k x}\right)\right) \psi\left(g\left(\vartheta^{k x}\right)\right)\right|^{2 \nu} \\
& \quad=L^{-2 \nu} N T^{2 \nu-2} \sum_{x \in \mathbb{Z}_{t}}\left|\sum_{k \in \mathcal{L}} \chi\left(f\left(\vartheta^{k x}\right)\right) \psi\left(g\left(\vartheta^{k x}\right)\right)\right|^{2 \nu} .
\end{aligned}
$$

Let $d=(p-1) / t$. Then for each power $\vartheta^{x}, x \in \mathbb{Z}_{t}$, there exist precisely $d$ values of $z \in \mathcal{U}_{p}$ such that $\vartheta^{x} \equiv z^{d}(\bmod p)$. Therefore,

$$
\begin{aligned}
& \sum_{x \in \mathbb{Z}_{t}}\left|\sum_{k \in \mathcal{L}} \chi\left(f\left(\vartheta^{k x}\right)\right) \psi\left(g\left(\vartheta^{k x}\right)\right)\right|^{2 \nu} \\
&= d^{-1} \sum_{z \in \mathcal{U}_{p}}\left|\sum_{k \in \mathcal{L}} \chi\left(f\left(z^{d k}\right)\right) \psi\left(g\left(z^{d k}\right)\right)\right|^{2 \nu} \\
& \leq d^{-1} \sum_{z \in \mathbb{Z}_{p}}\left|\sum_{k \in \mathcal{L}} \chi\left(f\left(z^{d k}\right)\right) \psi\left(g\left(z^{d k}\right)\right)\right|^{2 \nu} \\
& \leq d^{-1} \sum_{j_{1}, \ldots, j_{\nu} \in \mathcal{L}} \sum_{k_{1}, \ldots, k_{\nu} \in \mathcal{L}}\left(\sum_{z \in \mathbb{Z}_{p}} \chi\left(\prod_{i=1}^{\nu} \frac{f\left(z^{d j_{i}}\right)}{f\left(z^{d k_{i}}\right)}\right) \psi\left(\sum_{i=1}^{\nu}\left(g\left(z^{d j_{i}}\right)-g\left(z^{d k_{i}}\right)\right)\right)\right. \\
& \quad \sum_{j_{1}, \ldots, j_{\nu} \in \mathcal{L}} \sum_{k_{1}, \ldots, k_{\nu} \in \mathcal{L}}\left(\sum_{z \in \mathbb{Z}_{p}} \chi\left(\prod_{i=1}^{\nu} \frac{f\left(z^{d r j_{i}}\right)}{f\left(z^{d r k_{i}}\right)}\right) \psi\left(\sum_{i=1}^{\nu}\left(g\left(z^{d r j_{i}}\right)-g\left(z^{d r k_{i}}\right)\right)\right),\right.
\end{aligned}
$$

because $\operatorname{gcd}(r, t)=1$.

We need to bound the inner sum. For those configurations where each of the integers $\left(k_{1}, \ldots, k_{\nu}, j_{1}, \ldots, j_{\nu}\right)$ occurs at least twice, we shall use the trivial bound. This gives a contribution of $O\left(L^{\nu} p\right)$.

The generic situation, where the above does not occur, trivially happens in at most $L^{2 \nu}$ ways, and for each of these terms we wish to apply Lemma 3 In the case where we assume the additive character acts non-trivially that bound automatically applies due to our assumptions about $\psi$ and $g$, since in particular we know that $\left(k_{1}, \ldots, k_{\nu}\right)$ is not a permutation of $\left(j_{1}, \ldots, j_{\nu}\right)$. 
In the remaining case the multiplicative character acts non-trivially, due to our assumptions about $\chi$ and $f$. Here, because we know that at least one of the integers $k_{i}, j_{i}$ occurs with multiplicity one in the configuration, it follows from Lemma 1 that Lemma 3 applies.

Thus, in either case, each such term gives a contribution of $O\left(D d h p^{1 / 2}\right)$. Hence

$$
\begin{aligned}
\left|S_{\mathcal{Z}}(\chi, \psi ; f, g)\right|^{2 \nu} & \ll L^{-2 \nu} d^{-1} N T^{2 \nu-2}\left(L^{\nu} p+L^{2 \nu} D d h p^{1 / 2}\right) \\
& \ll N T^{2 \nu-2}\left(L^{-\nu} t+D h p^{1 / 2}\right) .
\end{aligned}
$$

Therefore $S_{\mathcal{Z}}(\chi, \psi ; f, g) \ll N^{1 / 2 \nu} T^{1-1 / \nu}\left(L^{-1 / 2} t^{1 / 2 \nu}+h^{1 / 2 \nu} D^{1 / 2 \nu} p^{1 / 4 \nu}\right)$. Basic manipulations and Lemma 2 yield the desired result.

\section{Applications}

There are many examples of specific sequences $\mathcal{Z}$ to which Theorem 4 applies. We provide bounds for the same sequences considered in [2]. In particular, for an integer $n \geq 2$ and $\lambda \in \mathcal{U}_{t}$ of multiplicative order $T$ modulo $t$, we define

$$
\begin{aligned}
& S_{n}(\chi, \psi ; f, g)=\sum_{x \in \mathbb{Z}_{t}} \chi\left(f\left(\vartheta^{x^{n}}\right)\right) \psi\left(g\left(\vartheta^{x^{n}}\right)\right), \\
& S_{n}^{*}(\chi, \psi ; f, g)=\sum_{x \in \mathcal{U}_{t}} \chi\left(f\left(\vartheta^{x^{n}}\right)\right) \psi\left(g\left(\vartheta^{x^{n}}\right)\right), \\
& U_{\lambda}(\chi, \psi ; f, g)=\sum_{x=1}^{T} \chi\left(f\left(\vartheta^{\lambda^{x}}\right)\right) \psi\left(g\left(\vartheta^{\lambda^{x}}\right)\right) .
\end{aligned}
$$

For these sequences one now derives from Theorem 4 complete analogues of Theorems 6, 7 and 8 of 2 which for the sake of completeness we now formulate in the following form.

Theorem 5. Let $f(X)$ and $g(X)$ be two polynomials over $\mathbb{F}_{p}$ each of degree at most $D$. Assume that at least one of the following holds:

- either the multiplicative character $\chi$ is non-trivial of order $s$ and $f$ is not an $s$-th power of a rational function over the algebraic closure of $\mathbb{F}_{p}$;

- or $\psi$ is non-trivial and $g$ is not constant modulo $p$.

Let $n \geq 2$ and let $\lambda \in \mathcal{U}_{t}$ be of multiplicative order $T$ modulo $t$. Then for any integer $\nu \geq 1$, the following bounds hold:

$$
\begin{aligned}
& S_{n}(\chi, \psi ; f, g) \ll t^{3 / 4} p^{1 / 8+\varepsilon}, \\
& S_{n}^{*}(\chi, \psi ; f, g) \ll \begin{cases}t^{3 / 4} p^{1 / 8+\varepsilon}, & \text { if } n=2 \text { or } t \text { is cube-free }, \\
t^{1-\alpha(n)} p^{\alpha(n) / 2+\varepsilon}, & \text { otherwise },\end{cases} \\
& U_{\lambda}(\chi, \psi ; f, g) \ll T^{1-(2 \nu+1) / 2 \nu(\nu+1)} t^{1 / 2 \nu} p^{1 / 4(\nu+1)},
\end{aligned}
$$

where $\alpha(n)$ is given by

$$
\alpha(n)=\frac{\lceil n / 2\rceil-1}{n\lceil n / 2\rceil+1}
$$

and the implied constant depends on $\varepsilon$ and $\nu$. 


\section{REFERENCES}

[1] M. C. R. Butler, 'The irreducible factors of $f\left(x^{m}\right)$ over a finite field', J. London Math. Soc., 30 (1955), 480-482. MR0071463 (17,130d)

[2] J. B. Friedlander, J. Hansen, and I. E. Shparlinski, 'On character sums with exponential functions', Mathematika, 47 (2000), 75-85. MR.1924489 (2003g:11089)

[3] J. B. Friedlander, S. V. Konyagin and I. E. Shparlinski, 'Some doubly exponential sums over $\mathbb{Z}_{m}$ ', Acta Arith., 105 (2002), 349-370. MR.1932568 (2004c:11147)

[4] W. C. W. Li, Number Theory with Applications, World Scientific Publishing Co., Singapore, 1996. MR:1390759 (98b:11001)

[5] R. Lidl and H. Niederreiter, Finite Fields, Cambridge University Press, Cambridge, 1997. MR $1429394(97 i: 11115)$

Department of Mathematics, University of Glasgow, Glasgow G12 8QW, Scotland, UNITED KINGDOM

E-mail address: sdc@maths.gla.ac.uk

School of Mathematics and Statistics, Carleton University, Ottawa, Ontario, CANADA K1S 5B6

E-mail address: mdewar@magma.ca

Department of Mathematics, University of Toronto, Toronto, Ontario, Canada M5S $3 \mathrm{G} 3$

E-mail address: frdlndr@math.toronto.edu

School of Mathematics and Statistics, Carleton University, Ottawa, Ontario, CANADA K1S 5B6

E-mail address: daniel@math.carleton.ca

Department of Computing, Macquarie University, Sydney, NSW 2109, Australia

E-mail address: igor@ics.mq.edu.au 